\title{
The Influence of Surgery Opportunity Choice on the Prognosis of the Post-Necrotizing Enterocolitis Intestinal Obstruction
}

\author{
Jixue Zhao ${ }^{1, a}$ and Xin $\mathrm{Fu}^{2, \mathrm{~b}}$, * \\ ${ }^{1}$ Department of Pediatric Surgery, the First Hospital of Jilin University, Changchun 130021, China; \\ ${ }^{2}$ Nursing Administration Department, China-Japan Union Hospital of Jilin University, Changchun \\ 130033, China \\ a xinfu0431@126.com, bjixuezhao0431@126.com
}

Keywords: Necrotizing enterocolitis, Surgery opportunity choice, Post-necrotizing enterocolitis intestinal obstruction.

\begin{abstract}
Objective: To investigate the treatment effect of the different surgery opportunity choice on the post- necrotizing enterocolitis intestinal obstruction. Methods: A retrospective analysis of our Hospital in recent 8 years, neonatal and pediatric surgery in the diagnosis and treatment of the postnecrotizing enterocolitis intestinal obstruction cases. All the cases were divided into two groups: early operation group and non-early operation group. The cases of non-early operation group were treated conservatively, and would get surgical treatment when the intestinal obstruction occurs repeatedly or the intestinal obstruction recovers difficultly. The survival children were followed up to 6 months. Clinical data were retrospectively analyzed, including the hospital stays, the reoccurrence of intestinal obstruction, weight gain 2 month after discharge. Results: The hospital stays of the experimental group was obviously shorter than that of control group $(\mathrm{P}<0.05)$, the reoccurrence rate of intestinal obstruction of the experimental group was obviously lower than that of control group $(9.1 \%$ vs53.8\%) $(\mathrm{P}<0.05)$. And the weight gain 2 month after discharge of the experimental group was obviously more than that of control group $(\mathrm{P}<0.05)$. Conclusion: Early operation intervention can shorten hospital stay of post- NEC intestinal obstruction, reduce the later complications, and promote the recovery.
\end{abstract}

\section{Introduction}

Necrotizing enterocolitis (NEC) is a severe complication frequently seen during the neonatal period. It is an inflammatory disease process of the gastrointestinal tract commonly seen in the neonatal unit affecting mostly premature infants $[1,2]$. NEC developes rapidly, and has high fatality rate and more complications. The main pathological characteristics of NEC is the extensive hemorrhage necrosis of small intestine and the colon. NEC has higher morbidity and mortality rates among the premature and low birth weight $[3,4]$.

Many NEC cases which have no intestinal necrosis and perforation can get conservative treatment during early stage, and many of them could get successful treatment which has the cure rate of $82.1 \%[2]$. But there exist a most common complication among the post- necrotizing enterocolitis cases that is intestinal obstruction. Post- NEC intestinal obstruction always trouble the doctors. There is a big controversy between the early operation and the conservative treatment. According to our experience, conservative treatment last long and the recovery was slow. Maybe early operation intervention can improve the condition rapidly and promote the recovery. Based on these considerations above, From August of 2009, we try to perform early operation for the post- NEC intestinal obstruction cases, and we have achived desirable effect.

\section{Patients and Methods}

\subsection{Patients}

From January of 2009 to January of 2017, 48 infants with post-necrotizing enterocolitis intestinal obstruction in the neonatology department, the First Hospital of Jilin University were enrolled in this 
retrospective analysis. Each patient signed an informed consent form. Approval was obtained from the institutional review committee of Jilin University.

\subsection{Study Design}

The patients were divided into two groups randomly: early operation group (experimental group) and non-early operation group (control group). The cases of non-early operation group were treated conservatively, and would get surgical treatment when the intestinal obstruction occurs repeatedly or the intestinal obstruction recovers difficultly. The survival children were followed up to 6 months.

We use the frequent operation methods, such as one-stage resection and anastomasis, enterolysis.

\subsection{Evaluation Criteria of Treatment Effect}

Evaluation criteria included the survival rate and the incidence of incomplete intestinal obstruction.

\subsection{Statistical Analysis}

All measured parameters including the the hospital stays, the reoccurrence of intestinal obstruction, weight gain after discharge were analyzed by the statistical software program Statistical Product and Service Solutions (SPSS) 17.0 (SPSS Inc., Chicago, IL, USA) and expressed as mean \pm standard deviation $( \pm \mathrm{s})$, and t-test was used. Enumeration data including gender, prevalence frequency were analyzed by $\chi 2$ test. $\mathrm{P}<0.05$ was considered significant.

\section{Results}

\subsection{Baseline Characteristic}

There were no significant difference $(\mathrm{P}>0.05)$ in general data including patients age, gender between the two groups (Table 1.).

Table 1. Baseline characteristics of study patients

\begin{tabular}{cccc}
\hline Characteristic & control group(26 cases) & experimental group (22 cases) & $P$ value \\
\hline Patients age (days) & $32.1 \pm 5.9$ & $29.8 \pm 6.6$ & non-significant \\
Female & 10 & 8 & non-significant \\
Male & 16 & 14 & non-significant \\
\hline
\end{tabular}

\subsection{Evaluation of Treatment Effects}

Among the cases of the early operation group (experimental group), all the 22 cases recovered after the operation, and the average length of stay is 10.6 days. Among the 26 cases of the non-early operation group(control group), 10 cases was discharged with a better health condition after the conservative treatment, 16 cases were performed operation after the long-term no effective conservative treatment or repeated intestinal obstruction, and the average length of stay is 22.6 days. The hospital stays of the experimental group was obviously shorter than that of control group $(\mathrm{P}<0.05)$, the reoccurrence rate of intestinal obstruction of the experimental group was obviously lower than that of control group $(9.1 \% \mathrm{vs} 53.8 \%)(\mathrm{P}<0.05)$. And the weight gain 2 month after discharge of the experimental group was obviously more than that of control group $(\mathrm{P}<0.05)$, as is shown in Table 2. Postoperative follow-up was conducted for 6 months.

Table 2 Efficacy of the two groups

\begin{tabular}{cccc}
\hline Characteristic & control group(26 cases) & experimental group(22 cases) & $P$ value \\
\hline Hospital stays (days) & $11.5 \pm 2.3$ & $23.4 \pm 4.1$ & $P<0.05$ \\
Weight gain 2 month after discharge $(\mathrm{kg})$ & $0.42 \pm 0.05$ & $1.01 \pm 0.21$ & $P<0.05$ \\
The reoccurrence rate of intestinal obstruction & $14 / 26$ & $2 / 22$ & $P<0.05$ \\
\hline
\end{tabular}

\section{Discussion}

Necrotizing enterocolitis (NEC) is one of the most common gastrointestinal tract disease frequently affecting premature infant with an incidence of $3 \%$ to $5 \%$, depending on the gestational 
age (GA) of infant at birth[5,6] and mortality rate of as high as 50\% [3,4]. Most cases of NEC are managed conservatively, with optimum medical treatment, bowel rest, abdominal decompression, parenteral nutrition, as well as antibiotics treatment, and recovered early.

But post- NEC intestinal obstruction is one of the common and inevitable complications. The main reason of post- NEC intestinal obstruction is the intestinal stricture, other reason including adhesion, poor peristalsis, and so on. Stricture is a well-known common complication of NEC during posthealing process, affecting about $20 \%$ of NEC survivors [7]. After the acute episode of NEC, PostNEC intestinal obstruction develop at a variable period irrespective of the mode of management, which is associated with severe and prolonged morbidity (septicemia, perforation, intestinal obstruction) and morbidity secondary to intestinal strictures [8] .The ischemic injury to the inner muscular layer of the intestine plays an important role in the pathogenesis of NEC [9]. For the treatment of post- NEC intestinal obstruction, at present, conservative treatment is always recommended, including gastrointestinal decompression, purge enema, abdominal physical therapy, and so on. However, in many ways, these conservative treatmets were difficult to achieve the desired effect. Many cases would perform the laparotomy after long-term conservative treatmet. And long-term conservative treatment of post-NEC intestinal obstruction will not only increase the workload of the clinican, but also increase the patient's pain and economic burden.

Based on these considerations above, we try to perform early operation for the post-NEC intestinal obstruction cases to take place of the operation after long-term conservertive treatment. According to our experience, early operation can shorten the hospital stays obviously, and increase the weight gain 2 month after discharge obviously. And at the same time, the early operation could decrease the reoccurrence rate of intestinal obstruction.

Early operation is better for the post-NEC intestinal obstruction, we analyze the possible reason: on the one hand, for the post-NEC intestinal obstruction, whether it is intestinal stricture or adhesion, the operation can relive the obstruction and restore the intestinal tract, and restore the digestion-absorption function, promote the early recovery. On the other hand, the early cases were always in comparative good condition, without electrolyte disturbance, malnutrition, so they can recover rapidly with less complications.

Post-NEC intestinal obstruction is a well-known complication of the healing process that follows ischaemic injury to the inner muscular layer of the intestine, during the acute episode. It is important fot the NEC treatment to explore the appropriate method to cope with the post-NEC intestinal obstruction. In our opinion, early operation intervention were more beneficial to the post-NEC intestinal obstruction than the operation after long-term conservative treatment.

\section{Summary}

Early operation intervention can shorten the hospital stays obviously, increase the weight gain 2 month after discharge obviously, and decrease the reoccurrence rate of intestinal obstruction obviously. And promote the early recovery. So the early operation for the post-NEC intestinal obstruction is feasible in clinic.

\section{References}

[1]. Kim SS, Albanese CT. Necrotizing enterocolitis. In: Grosfeld JL, O’Neill JA, Fonkalsrud EW, Coran AG (eds). Pediatric Surgery. 6th edn. Philadelphia: Mosby/Elsevier, 2006, pp 1427-52

[2]. Henry MCW, Moss RL. Necrotizing enterocolitis. In: Stringer MD, Oldham KT, Mouriquand PDE (eds). Pediatric Surgery and Urology Long-term Outcomes. 2nd edn. Cambridge: Cambridge University Press, 2006, pp 329-50.

[3]. Moss RL, Kalish LA, Duggan C, et al. Clinical parameters do not adequately predict outcome in necrotizing enterocolitis: a multiinstitutional study. J Perinatol 2008; 28:665-74. 
[4]. Holman RC, Stoll BJ, Curns AT, et al. Necrotising enterocolitis hospitalisations among neonates in the United States. Paediatr Perinat Epidemiol 2006; 20:498-506.

[5]. Houben $\mathrm{CH}$, Chan KW, Mou JW, et al. Management of intestinal strictures post conservative treatment of necrotizing enterocolitis: the long term outcome. J Neonatal Surg 2016; 5:28.

[6]. Heida FH, Loos MH, Stolwijk L, et al. Risk factors associated with postnecrotizing enterocolitis strictures in infants. J Pediatr Surg 2016; 51:1126-30.

[7]. Schwartz MZ, Hayden CK, Richardson CJ, et al. A prospective evaluation of intestinal stenosis following necrotizing enterocolitis. J Pediatr Surg 1982; 17:764-70.

[8]. Blakely ML, Lally KP, McDonald S, et al. NEC Subcommittee of the NICHD Neonatal Research Network. Postoperative outcomes of extremely low birth-weight infants with necrotizing enterocolitis or isolated intestinal perforation: a prospective cohort study by the NICHD Neonatal Research Network. Ann Surg 2005; 241:984-9.

[9]. Crafts TD, Hunsberger EB, Jensen AR, et al. Direct peritoneal resuscitation improves survival and decreases inflammation after intestinal ischemia and reperfusion injury. J Surg Res 2015; 199: 428-34. 